\title{
Hinweise für die Benutzung des Löwe-Rosenberg
}

\section{Inhalt der Kommentierung}

Der LÖWE-RosEnBERG kommentiert die StPO, das EGStPO, das GVG und das EGGVG mit Ausnahme der nur den Zivilprozess betreffenden Teile, sowie - mit dem Schwerpunkt auf den strafverfahrensrechtlich besonders bedeutsamen Regelungen - die EMRK und den IPBPR. Wenig bekannte oder schwer auffindbare strafverfahrensrechtliche Nebengesetze, deren Wortlaut für die Kommentierung erforderlich ist, werden bei den einschlägigen Erläuterungen im Kleindruck wiedergegeben.

\section{Erscheinungsweise und Stand der Bearbeitung}

Die 27. Auflage des LöWE-ROSENBERG erscheint in Bänden, deren ErscheinungsReihenfolge von der des Gesetzes abweichen kann. Die Bände werden in der vom Gesetz vorgegebenen Reihenfolge durchnumeriert.

Der Stand der Bearbeitung ist dem Vorwort jedes Bandes zu entnehmen. Die Autoren sind bemüht, besonders wichtige Änderungen und Entwicklungen auch noch nach diesem Stichtag bis zur Drucklegung des Bandes zu berücksichtigen.

\section{Bearbeiter}

Jeder Bearbeiter (in der Fußzeile angegeben) trägt für seinen Teil die alleinige inhaltliche Verantwortung. Die Stellungnahmen zu Rechtsfragen, die an mehreren Stellen des Kommentars behandelt werden, können daher voneinander abweichen. Auf solche Abweichungen wird nach Möglichkeit hingewiesen.

\section{Aufbau der Kommentierung}

Neben der umfassenden Einleitung zum Gesamtwerk sind den Untereinheiten der kommentierten Gesetze (Bücher, Abschnitte, Titel), soweit erforderlich, Vorbemerkungen vorangestellt, die das für die jeweilige Untereinheit Gemeinsame erläutern.

Der den Vorbemerkungen und den Kommentierungen der einzelnen Vorschriften erforderlichenfalls vorangestellte Abschnitt Geltungsbereich enthält Hinweise auf zeitliche und örtliche Besonderheiten. Der Abschnitt Entstehungsgeschichte gibt, abgesehen von ganz unwesentlichen Änderungen, die Entwicklung der geltenden Fassung der Vorschrift vom Erlass des jeweiligen Gesetzes an wieder. Fehlt er, so kann davon ausgegangen werden, dass die Vorschrift unverändert ist. Der Hinweis auf geplante Änderungen verzeichnet Änderungsvorschläge, die sich beim Abschlusszeitpunkt der Lieferung im parlamentarischen Gesetzgebungsverfahren befinden.

Die Erläuterungen sind nach systematischen Gesichtspunkten gegliedert, die durch Überschriften oder Stichworte hervorgehoben sind. In der Regel ist den Erläuterungen eine systematische Übersicht vorangestellt. Soweit angebracht wird sie bei besonders umfangreichen Erläuterungen durch eine alphabetische Übersicht ergänzt. Bei den Erläuterungen selbst werden für jede Vorschrift (zur Erleichterung des Zitierens) durchlaufende Randnummern verwendet. 


\section{Schrifttum}

Der Kommentar enthält am Anfang jedes Bandes ein allgemeines Literaturverzeichnis, das nur die häufiger verwendete oder allgemeine Literatur enthält. Den Vorbemerkungen und den Kommentierungen der einzelnen Vorschriften sind Schrifttumsverzeichnisse vorangestellt, die einen Überblick über das wesentliche Schrifttum zu dem jeweils behandelten Thema geben.

\section{Zitierweise}

Literatur, die in diesen Schrifttumsverzeichnissen enthalten ist, wird im laufenden Text im allgemeinen nur mit dem Namen des Verfassers (ggfs. mit einer unterscheidenden Kurzbezeichnung) oder der sonstigen im Schrifttumsverzeichnis angegebenen Kurzbezeichnung zitiert, doch wird bei Veröffentlichungen in Zeitschriften vielfach auch die genaue Fundstelle nachgewiesen. Sonst sind selbständige Werke mit (gelegentlich verkürztem) Titel und Jahreszahl, unselbständige Veröffentlichungen (auch Beiträge in Festschriften u.ä.) mit der Fundstelle angegeben. Auflagen sind durch hochgestellte Zahlen gekennzeichnet; fehlt eine solche Angabe, so wird aus der Auflage zitiert, die im allgemeinen Schrifttumsverzeichnis angegeben ist. Hat ein Werk Randnummern, so wird nach diesen, sonst nach Seitenzahl oder Gliederungspunkten zitiert.

Befindet sich beim Zitat anderer Kommentare die in Bezug genommene Stelle im gleichen Paragraphen, so wird nur die Randnummer oder (bei deren Fehlen) der Gliederungspunkt angegeben; wird auf die Erläuterungen bei einem anderen Paragraphen Bezug genommen, so wird dieser genannt. Entsprechend wird auch im LöWE-ROSENBERG selbst verwiesen. Bei diesem wird, wenn nichts anderes angegeben ist, auf die gegenwärtige 27. Auflage verwiesen. Ist der Band mit den Erläuterungen, auf die verwiesen werden soll, noch nicht erschienen, so ist, soweit dies sachdienlich erschien, in Klammern ergänzend die genaue Fundstelle in der 26. Auflage angegeben.

Zeitschriften werden regelmäßig mit dem Jahrgang zitiert. Ausnahmen (Bandangabe) bilden namentlich ZStW, GA (bis 1933) und VRS; hier ist regelmäßig die Jahreszahl zusätzlich angegeben. Bei der Angabe der Fundstelle eines amtlichen Verkündungsblattes wird die Jahreszahl nur angegeben, wenn sie von der Jahreszahl der Rechtsvorschrift abweicht.

Entscheidungen werden im allgemeinen nur mit einer Fundstelle angegeben. Dabei hat die amtliche Sammlung eines obersten Bundesgerichtes den Vorrang, sonst die Fundstelle, die die Entscheidung mit Anmerkung oder am ausführlichsten wiedergibt.

\section{Abkürzungen}

Die verwendeten Abkürzungen, namentlich von Gesetzen, Verwaltungsvorschriften, Entscheidungssammlungen, Zeitschriften usw. sind im Abkürzungsverzeichnis nachgewiesen. 